\title{
OPTICAL-TO-SAR IMAGE REGISTRATION BASED ON GAUSSIAN MIXTURE MODEL
}

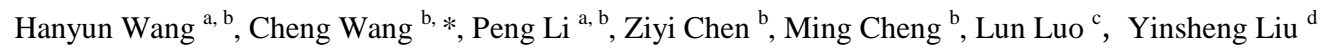 \\ ${ }^{a}$ School of Electronic Science and Engineering, National University of Defense Technology, Changsha, China - \\ hanyun.wang1986@gmail.com,peng.li.scholar@gmail.com \\ ${ }^{\mathrm{b}}$ Department of Computer Science, School of Information Science and Technology, Xiamen University, Xiamen, China \\ - cwang@xmu.edu.cn, chenziyicip@gmail.com \\ c China Transport Telecommunication \& information Center, Beijing, China \\ d Hunan Provincial Transport Technical Information Center, Changsha, China
}

KEY WORDS: Remote Sensing, Image Registration, SAR, Optical, Gaussian Mixture Model, EM Algorithm, Line Support Region

\begin{abstract}
:
Image registration is a fundamental in remote sensing applications such as inter-calibration and image fusion. Compared to other multi sensor image registration problems such as optical-to-IR, the registration for SAR and optical images has its specials. Firstly, the radiometric and geometric characteristics are different between SAR and optical images. Secondly, the feature extraction methods are heavily suffered with the speckle in SAR images. Thirdly, the structural information is more useful than the point features such as corners. In this work, we proposed a novel Gaussian Mixture Model (GMM) based Optical-to-SAR image registration algorithm. The feature of line support region (LSR) is used to describe the structural information and the orientation attributes are added into the GMM to avoid Expectation Maximization (EM) algorithm falling into local extremum in feature sets matching phase. Through the experiments it proves that our algorithm is very robust for optical-to- SAR image registration problem.
\end{abstract}

\section{INTRODUCTION}

In many image processing applications it is necessary to compare multiple images of the same scene acquired by different sensors such as inter-calibration of multi sensors and image fusions. But because of the differences of radiometric and geometric characteristics, registration becomes more difficult and complex, especially for SAR and optical images.

Many works have been down about optical-to-SAR image registration problem. According to the method reviewed in the literature [1], the registration methods can be divided into two classes; intensity-based method and feature-based method. The methods based on image intensity directly are not suitable for optical-to-SAR image registration because of the noteworthy differences of intensity characteristics between optical and SAR images. Mutual information (MI) is a state-of-art method in multi sensor image registration. Original MI algorithm is an intensity-based method and has a big shortcoming in incorporating spatial information. Some improved methods incorporated spatial information such as gradient magnitude, gradient orientation and histogram combined edge orientation with intensity have been proposed and used for multi sensor image registration[2][3][4][5][6]. A mutual information method is proposed for TerraSAR-X and Ikonos images registration in Urban Areas [7]. Pluim has done a good survey on multi-model image registration based on mutual information [8]. Mahmudul extends an information theoretic measure called the crosscumulative residual entropy (CCRE) for optical-to-SAR image registration [10]. A similarity metric and a classification method are used to improve the metric registration in [11]. Compared to intensity-based methods, the feature-based approaches are more flexible, this is because that the information used in matching is basically the structural information in the images [9] [12]. A new Gaussian Mixture Model based multi-model image registration method is proposed in [13], but only the spatial information is used in the model. Features such as edge and contour are more suitable for describing structure than the intensity values. However, the feature-based approaches are often suffered from three aspects: first, the extraction of the complex features like line segment or unique shape are not reliable; second, the matching procedure are often corrupted by the change of the structures and noise in the images. Especially for the optical-to-SAR image registration, the feature extraction and matching is a more complex and difficult problem because of the speckle in SAR images.

In this work, we proposed a novel Gaussian Mixture Model (GMM) based Optical-to-SAR image registration algorithm. The feature of line support region (LSR) is proposed to describe the structural information and an improved Gaussian Mixture Model incorporated both spatial coordinates and gradient orientation information is used for feature point sets registration in the feature matching phase. LSR is a feature point set grouped by similar edge orientation. GMM is a probabilistic model easy to consider the existence of outliers, noises and missing points. The parameters are estimated through maximizing the posterior probability with EM algorithm.

The rest of the paper is organized as follows. In section 2, the feature extraction algorithm of line support region (LSR) is given. In section 3, we first formulate the basis GMM point set registration framework, and then give a derivation of attributestrengthened GMM framework in detail when other attributes are added into. In the last of this section, the orientationstrengthened GMM framework used for Optical to SAR image registration is introduced. The experiments are discussed in section 4. Section 5 concludes that our method is useful and robust for Optical-to-SAR image registration.

\footnotetext{
* Corresponding author. This is useful to know for communication with the appropriate person in cases with more than one author.
} 


\section{LSR EXTRACTION}

LSR is originally the semi-finished product of the phase-based line segment extraction, and is more stable than line features. We use the phase-based line extraction frame as the basic frame for the extraction of LSR [14]. The frame groups the connected edge points that have similar edge orientation. Figure 1 illustrates the basic idea with a gray level image (Figure 1. (a)). Two steps are involved:

(1) Detect the edge points and calculate their orientation (Figure 1. (b)). The edge orientation is quantified into several orientations, for instance, 8 orientations. The non-edge pixels are signed as background.

(2) Group the edge points. Connected edge points that have the same orientation are grouped into the same set (Figure 1. (c)), and the set is called a line support region (LSR). If it is to extract the line segments in the image, then every LSR can be used as a sample set to fit a line segment (Figure 1. (d)).

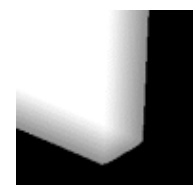

(a)

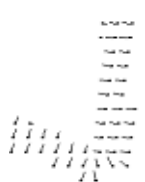

(b)

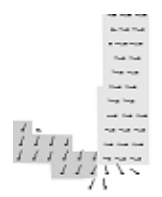

(c)

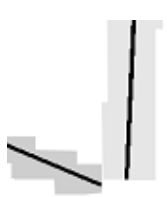

(d)
Figure 1. Phase-based LSR extraction. (a) Original image. (b)

Edge phase. (c) Phase-based grouping. (d) LSR and lines.

The grouping step can be described as the classical imagelabelling problem: mark the connected equivalent pixels with the same labels and the disconnected sets are marked with different labels. In this case the "equivalent" means same in quantified edge orientation. What is need to consider is that in this case the qualification of the orientation may cause serious break of the LSRs, and this can be solved with the overlapping method [14]. In order to filter out the scrappy structures, an individual LSR must cover enough pixels; otherwise it will be sign as background.

LSR has several merits: first, it reflects the structures and it is more stable than line feature or shape feature; second, the qualified edge orientation signed inside LSR is not sensitive to the noise.

\section{GMM PROBABILISTIC FRAMEWORK FOR FEATURE POINT SETS REGISTRATION}

\subsection{GMM Point Sets Registration Framework}

In the Gaussian Mixture Model point set framework [15][18], the two point sets are related by GMM probability density function, that is to say one point set represents the Gaussian Mixture Model (GMM) centroids and the other one represents the observed points. Throughout the paper we use the following notations:

- D - dimension of the point set;

- N,M - number of the points in the point sets;

- $\mathrm{X}_{\mathrm{N} * \mathrm{D}}=\left(\mathrm{x}_{1}, \ldots \mathrm{X}_{\mathrm{N}}\right)^{\mathrm{T}}$ - the first point set (the observed points);

$\mathrm{Y}_{\mathrm{M}^{*} \mathrm{D}}=\left(\mathrm{y}_{1}, \ldots \mathrm{y}_{\mathrm{M}}\right)^{\mathrm{T}}$ - the second point set (the GMM centroids);

$\mathrm{T}(\mathrm{Y}, \theta)$ - Transformation $\mathrm{T}$ applied to $\mathrm{Y}$, where $\theta$ is a set of the transformation parameters;

I- identity matrix;
- 1- column vector of all ones;

- $\quad d(a)$ - diagonal matrix formed from the vector a; In the framework, the GMM probability density function is

$$
\begin{aligned}
& p(x)=\sum_{m=1}^{M} P(m) p(x \mid m) \\
& p(x \mid m)=\frac{1}{\left(2 \pi \sigma_{\mathrm{m}}{ }^{2}\right)^{D / 2}} \exp \\
& -\frac{\left\|x-y_{m}\right\|^{2}}{2 \sigma_{\mathrm{m}}^{2}}
\end{aligned}
$$

Without prior information each component has the same weight and each Gaussian is spherical i.e. the covariance is proportional to identity matrix. Here all components $(\mathrm{m}=1 \ldots \mathrm{M})$ of GMM use equal isotropic covariance $\sigma^{2}$ and equal member ship probabilities $\mathrm{P}(\mathrm{m})=1 / \mathrm{M}$. The noise and outliers are also considered as an additional uniform distribution $\mathrm{P}(\mathrm{x} \mid \mathrm{M}+1)=1 / \mathrm{N}$. Denoting the weight of the uniform distribution as $\mathrm{w}, 0 \leqq \mathrm{w} \leqq 1$, the mixture model takes the form

$$
p(x)=w \frac{1}{N}+(1-w) \sum_{m=1}^{M} \frac{1}{M} p(x \mid m)
$$

Our point set registration framework is based on the Myronenko's work [15]. Core to his method (called CPD) is force GMM centroid to move coherently as a group, which preserves the topological structure of the point sets and then can get a closed form solution to the maximization step of the EM algorithm for a general multi-dimensional case. The transformation parameters are estimated by maximizing the likelihood or equivalently by minimizing the negative loglikelihood function. The correspondence probability between two points $y_{m}$ and $x_{n}$ is the posterior probability of the GMM centroid given the data point:

$$
p\left(m \mid x_{n}\right)=P(m) p\left(x_{n} \mid m\right) / p\left(x_{n}\right)
$$

But what we should be concerned is that EM algorithm always requires a good initialization and is easy to fall into local extreme during the optimization process. In order to relax the requirements of good initialization and avoid getting a local solution, here we propose an Attribute-strengthened CPD algorithm in which the gradient orientation attributes are added into the Gaussian Mixture Model framework and can also get a global closed form solution using methodology of the CPD algorithm.

\subsection{Attribute-Strengthened GMM Framework}

Myronenko's work [15] based on Gaussian Mixture Model and EM algorithm only considers spatial localization information, and it easily falls into local extreme. In the first part of this section, we give a detailed formulation of the general framework about how the attributes are incorporated into the Gaussian Mixture Model framework and how we can get the closed form solution to the EM optimization method. In the 
next part of this section, we will introduce our method that both spatial localization information and gradient orientation information are incorporated into for optical-to-SAR image registration.

\subsubsection{The general framework}

First we only consider the point sets including two independent attributes which have the same weight for point sets registration and it is easy to generalize to multi attributes case. Define the following notations:

- $\quad \mathrm{a} b$ - independent attributes;

- $\mathrm{D}_{1}, \mathrm{D}_{2}$ - dimension of attribute $\mathrm{a}$ and $\mathrm{b}$ respectively;

- $\quad x_{n}=\left[a_{n 1}, \ldots a_{n D 1}, b_{n 1}, \ldots b_{n D 2}\right]=\left[a_{n}{ }^{T}, b_{n}{ }^{T}\right]^{T}$ - one element in point set $X$;

$y_{m}=\left[a_{m 1}, \ldots a_{m D 1}, b_{m 1}, \ldots b_{m D 2}\right]=\left[a_{m}{ }^{T}, b_{m}{ }^{T}\right]^{T}$ - one element in point set $\mathrm{Y}$;

Now we can define a new transformation formulation between the two point sets with two independent attributes as the following form:

$$
T\left(y_{m} ; B_{1}, B_{2}, t_{1}, t_{2}\right)=\left[\begin{array}{ll}
B_{1} & 0 \\
0 & B_{2}
\end{array}\right] y_{m}+\left[\begin{array}{l}
t_{1} \\
t_{2}
\end{array}\right]
$$

where $\mathrm{B}_{1(\mathrm{D} 1 * \mathrm{D} 1)}$ is a transformation matrix and $\mathrm{t}_{1(\mathrm{D} 1 * 1)}$ is a translation vector between $\mathrm{a}_{\mathrm{n}\left(\mathrm{D} 1 *_{1}\right)}$ and $\mathrm{a}_{\mathrm{m}\left(\mathrm{D} 1 *_{1}\right)}$. So does it for $\mathrm{B}_{2}$ and $t_{2}$. Substitute the equation (5) into the norm $\left\|\mathrm{x}_{\mathrm{n}}-\mathrm{T}\left(\mathrm{y}_{\mathrm{m}}, \theta\right)\right\|^{2}$, and it can be rewrite as

$$
\begin{aligned}
\| x_{n}- & T\left(y_{m}, \theta\right)\left\|^{2}=\right\| x_{n}-\left[\begin{array}{ll}
B_{1} & 0 \\
0 & B_{2}
\end{array}\right] y_{m}-\left[\begin{array}{l}
t_{1} \\
t_{2}
\end{array}\right] \|^{2} \\
= & \operatorname{tr}\left[\left(a_{n}-B_{1} a_{m}-t_{1}\right)^{T}\left(a_{n}-B_{1} a_{m}-t_{1}\right)\right] \\
& +\operatorname{tr}\left[\left(b_{n}-B_{2} b_{m}-t_{2}\right)^{T}\left(b_{n}-B_{2} b_{m}-t_{2}\right)\right]
\end{aligned}
$$

It can also be rewrite as the following form

$$
\begin{aligned}
\left\|x_{n}-T\left(y_{m}, \theta\right)\right\|^{2} & =\left\|a_{n}-B_{1} a_{m}-t_{1}\right\|^{2} \\
& +\left\|b_{n}-B_{2} b_{m}-t_{2}\right\|^{2}
\end{aligned}
$$

Now we can find that when there has two independent attributes, the displacement of two points coming from different point set is the sum of displacements of two corresponding attributes. Equation 7 is used to substitute the counterpart in the posterior probabilities of GMM components $\mathrm{P}\left(\mathrm{m} \mid \mathrm{x}_{\mathrm{n}}\right)$ and the EM objective function $\mathrm{Q}$. They now can be rewrite as

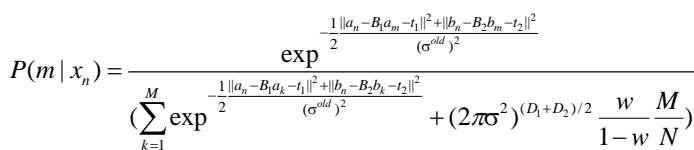

$$
\begin{aligned}
& Q\left(\theta, \sigma^{2}\right)=\frac{1}{2 \sigma^{2}} \sum_{n=1}^{N} \sum_{m=1}^{M} P\left(m \mid x_{n}\right)\left(\left\|a_{n}-B_{1} a_{m}-t_{1}\right\|^{2}+\left\|b_{n}-B_{2} b_{m}-t_{2}\right\|^{2}\right) \\
& +\frac{N_{P}\left(D_{1}+D_{2}\right)}{2} \log \sigma^{2}
\end{aligned}
$$

Now we can find it is the same form as the counterpart in the paper [15]. So we can get the closed form solution for Gaussian Mixture Model with two independent attributes too. For simplicity, we use deterministic annealing on it. We should notice that the transformation parameters $B_{1}$ and $t_{1}$ are independent of transformation parameters $B_{2}$ and $t_{2}$. All transformation parameters can be obtained through the methodology in the paper [15]. During the iteration of EM algorithm, we should re-parameterize all attributes using the new transformation parameters. The framework discussed above is easily generalized to three or more independent attributes and is useful for both rigid and non-rigid point set registration.

\subsubsection{Orientation-strengthened GMM framework}

In the context of Optical to SAR image registration, we propose an orientation-strengthened GMM algorithm for LSR feature sets registration. The two attributes used are spatial localization information and gradient orientation information. Because of different imaging mechanisms, the radiometric and geometric characteristics are different between SAR and optical images, the attributes based on gradient magnitude is not stable because of the speckle in SAR images. But gradient orientation information is a reflection of image structures and more stable than other attributes such as gradient magnitude information. Inherently gradient orientation is not completely independent of the spatial localization. But in this paper we assume that these two attributes are completely independent and through the experiments it proves that this relaxation is useful. Here we denote the spatial coordinates as attribute a (a $2 * 1$ vector) and gradient orientation as attribute $\mathrm{b}$ (a $1 * 1$ vector). That is to say that $\mathrm{D}_{1}=2, \mathrm{D}_{2}=1$.

The transformation matrix $\mathrm{B}_{1}$ and the translation vector $\mathrm{t}_{1}$ are the spatial transformation between two images. $B_{1}$ could be a rigid transformation matrix or affine transformation matrix. But in our method we assume that the transformation between optical and SAR images is rigid, and this hypothesis is suitable when the two remote sensing images has been systematically corrected. The transformation of gradient orientation between two remote sensing images according this hypothesis is only a translation, and the transformation matrix $\mathrm{B}_{2}$ must be an identity matrix. During the every iteration of EM algorithm, the centroid of GMM must be re-parameterized for both attributes to preserve the topological structure of the point sets. Through the experiments we find our method is more robust than original author's work and not easy to fall into local minimum. Compared to original algorithm the computation time is almost the same.

\section{EXPERIMENT RESULT}

We show the performance of our method on real optical and SAR image data. The stopping conditions for iterative process and initial value of $\mathrm{w}$ are the same as original value in the Myronenko's work[15]. The starting value $\sigma^{2}$ is 1 and gradually annealed with $\sigma^{2}=0.93$. All images have been preprocessed and all feature point sets are preprocessed to have zero mean and unit variance.

The experiments focus on the robustness of our modified framework than original CPD algorithm. The validity of the LSR in the optical to SAR image registration is demonstrated indirectly through the performance of registration results.

For the first experiment, we use a pair of optical and SAR images with relative simple structures. From Figure 2, we can 
find LSR features can represent the main structures of the image for both optical and SAR images. Compared Figure 3 (a) to Figure 3 (b), we can find that only using the spatial information is easy to fall into local extreme, but when the other attributes are added our algorithm will be more robust and not easy to fall into local extreme. For the second experiment (Figure 4, Figure 5), we use a pair of optical and SAR images with relative complex structures, and our method is also useful. The third experiment is given in Figure 6 and Figure 7.

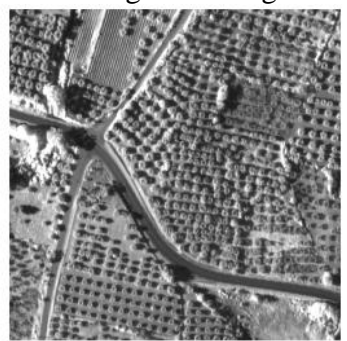

(a)

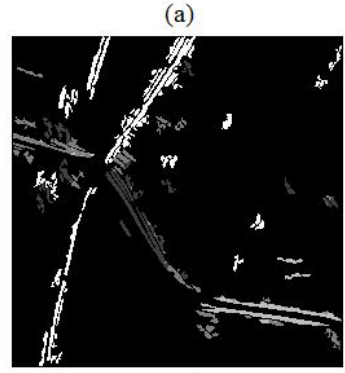

(c)

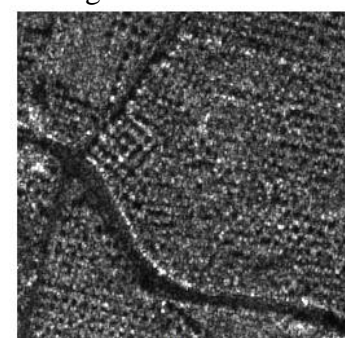

(b)

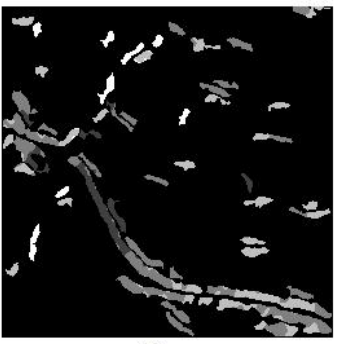

(d)

Figure 2. LSR extraction

(a) Optical image. (b) SAR image. (c) LSR of optical image. (d) LSR of SAR image.
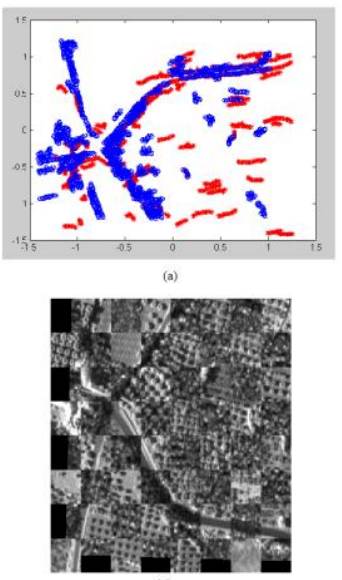

Figure 3 registration result

(a) Point set registration result of our method. (b) Point set registration result of CPD.(c) Image registration result of our method. (d) Image registration result of CPD.

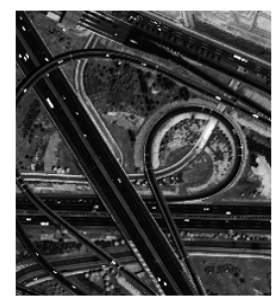

(a)

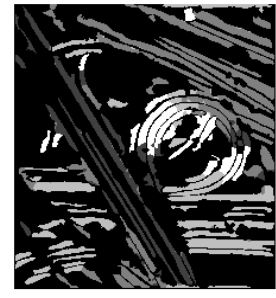

(c)

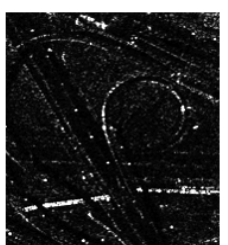

(b)

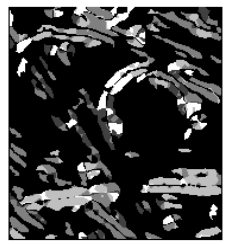

(d)
Figure 4 LSR extraction

(a) Optical image. (b) SAR image. (c). LSR of optical image (d) LSR of SAR image.
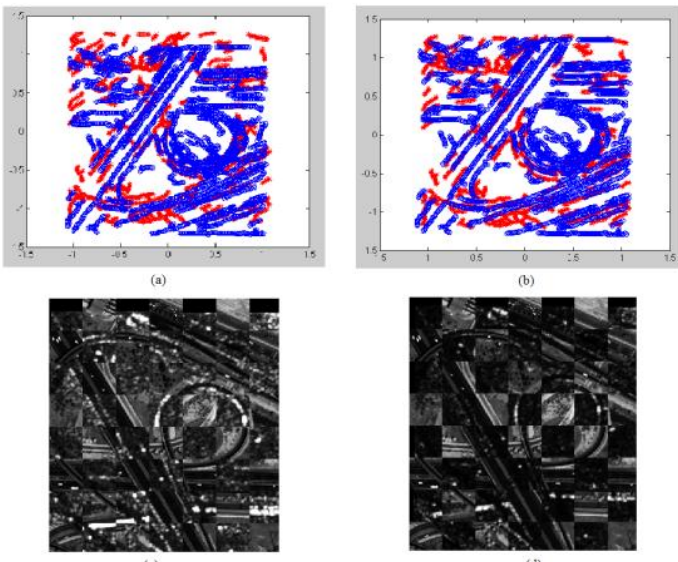

Figure 5 registration result

(a) Point set registration result of our method. (b) Point set registration result of CPD.(c) Image registration result of our method. (d) Image registration result of CPD.

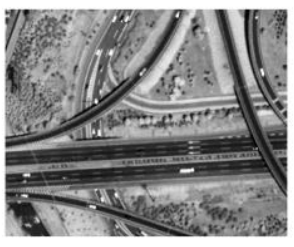

(a)

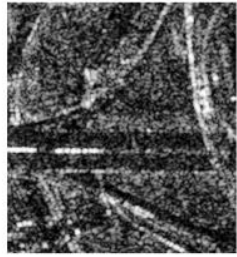

(c)

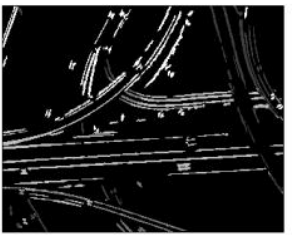

(b)

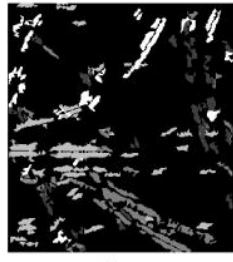

(d)
Figure 6 registration result

(a) Optical image. (b). LSR of optical image (c) SAR image. (d) LSR of SAR image. 

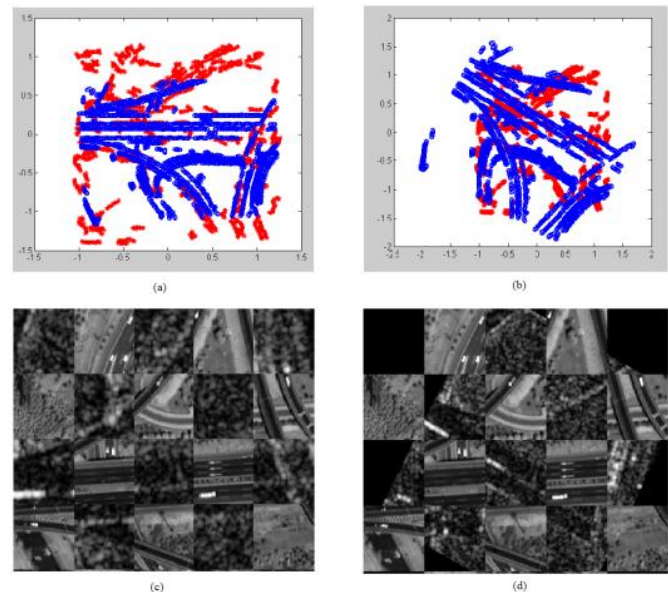

Figure 7 registration result

(a) Point set registration result of our method. (b) Point set registration result of CPD.(c) Image registration result of our method. (d) Image registration result of CPD.

\section{CONCLUSION}

In this paper, we proposed a novel Optical-to-SAR image registration algorithm based on LSR feature and Gaussian Mixture Model. LSR is a promising feature that can well describe the structures in both high resolution optical images and the SAR images with heavy noise. Gaussian Mixture Model is a robust statistic model for optical-to-SAR image registration. Proposed attributes added into GMM model can improve the robustness obviously when using the EM algorithm to estimate the transformation parameters. The orientation attribute is used and a corresponding model is given in the method but it is only suitable for rigid transformation. We can consider adding other attributes and suitable models for both rigid and non-rigid transformations. The proposed algorithm can also be used in inter-calibration of the optical and SAR sensors and other applications.

\section{REFERENCE}

[1] B. Zitová and J. Flusser, Image registration methods: A survey, Image and Vision Computing., vol. 21, no. 11, pp. 9771000, Oct. 2003.

[2] Josien P, Antoine M, Max V. Image Registration by Maximization of Combined Mutual Information and Gradient Information [J]. IEEE Transactions on Medical Imaging, 2000, 19(8):809-814.

[3] Wang Xiaoxiang, Tian Jie. Image Registration Based on Maximization of Gradient Code Mutual Information [J]. Image Analysis and Stereology, 2005, 24: 1-7.

[4] Shu Lixia, Tan Tieniu, Tang Ming, et al. A Novel Registration Method for SAR and SPOT Images[C]//Proc. of ICIP'05. Genova, Italy: [s. n.], 2005: 213-216.

[5] Kim Yong Sun, Lee Jae Hak, Ra Jong Beom. Multisensor Image Registration Based on Intensity and Edge Orientation Information [J]. Pattern Recognition, 2008, 41(11): 3356-3365.

[6] Shu Lixia, Tan Tieniu. SAR and SPOT Image Registration Based on Mutual Information with Contrast Measure[C]//Proc. of ICIP'07. San Antonio, USA: [s. n.], 2007: 429-432.

[7] Sahil Suri and Peter Reinartz. Mutual-Information-Based Registration of TerraSAR-X and Ikonos Imagery in Urban
Areas. IEEE Transactions On Geoscience And Remote Sensing, Vol. 48, No. 2, pp: 939-949, 2010.

[8] Pluim, J.P.W., J.B.A. Maintz, and M.A. Viergever. Mutual-Information-Based Registration of Medical Images: A Survey. IEEE Transaction On Medical Imaging, 2003. 22(8): p. 986-1004.

[9] Hui Li; Manjunath, B.S.; Mitra, S.K.. Optical-to-SAR image registration using the active contour model. Proceedings of the 27th Asilomar Conference on Signals, Systems and Computers, pp. 568-572, 0-8186-4120-7, Pacific Grove, CA, USA, November 01-03, 1993, IEEE, Computer Society Press, Los Alamitos, CA, USA.

[10] Mahmudul Hasan, Mark R. Pickering and Xiuping Jia. Multi-modal Registration of SAR and Optical Satellite Images. Digital Image Computing: Techniques and Applications, 2009: 447-453.

[11] J. Inglada and A. Giros. On the possibility of automatic multi sensor image registration, IEEE Trans. Geosci. Remote Sens., vol. 42, p. $2104,2004$.

[12] Lei Huang, Zhen Li; Rui Zhang. SAR and optical images registration using shape context. International conference on Geoscience and Remote Sensing Symposium., pp: 3556-3561, 2011.

[13] Peng Ye, Fang Liu. An Implicit Similarity Method for Multi-modal Image Registration. International Workshop on Multi-Platform/Multi-Sensor Remote Sensing and Mapping (M2RSM), 2011.

[14] B. BURNS. Extracting Straight Lines. IEEE Transactions on Pattern Analysis and Machine Intelligence, Volume 8, Issue 4, pp. 425-455, 1986.

[15] Myronenko, A., Song, X. Point-set registration: Coherent point drift. IEEE Transactions on Pattern Analysis and Machine Intelligence, Volume 32, Issue 12, pp. 2262-2275, 2010.

[16] A. Dempster, N. Laird, and D. Rubin. Maximum likelihood from incomplete data via the EM algorithm, Journal of Royal Statistical Society. Series B (Methodological), vol. 39, no. 1, pp. 1-38, 1977.

[17] C. M. Bishop, Neural Networks for Pattern Recognition. Oxford University Press, Inc., 1995.

[18] B. Jian and B.C. Vemuri. A robust algorithm for point set registration using mixture of Gaussians. ICCV, pages 1246$1251,2005$. 